\title{
Induction of protective immune responses against schistosomiasis using functionally active cysteine peptidases
}

\author{
Rashika El Ridi ${ }^{1}$, Hatem Tallima ${ }^{1}$, John P. Dalton ${ }^{2}{ }^{*}$ and Sheila Donnelly ${ }^{3}$ \\ ${ }^{1}$ Zoology Department, Faculty of Science, Cairo University, Cairo, Egypt \\ ${ }^{2}$ Medical Biology Centre, School of Biological Sciences, Queen's University Belfast, Belfast, Northern Ireland \\ ${ }^{3}$ The i-three Institute, University of Technology at Sydney, Ultimo, Sydney, NSW, Australia
}

\section{Edited by:}

Paul J. Brindley, The George Washington University, USA

\section{Reviewed by:}

Michel Drancourt, Université de la Méditerranée, France

David L. Bernick, University of California at Santa Cruz, USA

\section{${ }^{*}$ Correspondence:}

John P. Dalton, Medical Biology Centre, School of Biological Sciences, Queen's University Belfast, Belfast BT9 7BL, Northern Ireland e-mail: j.dalton@qub.ac.uk
Each year schistosomiasis afflicts up to 600 million people in 74 tropical and sub-tropical countries, predominantly in the developing world. Yet we depend on a single drug, praziquantel, for its treatment and control. There is no vaccine available but one is urgently needed especially since praziquantel-resistant parasites are likely to emerge at some time in the future. The disease is caused by several worm species of the genus Schistosoma. These express several classes of papain-like cysteine peptidases, cathepsins $B$ and $L$, in various tissues but particularly in their gastrodermis where they employ them as digestive enzymes. We have shown that sub-cutaneous injection of recombinant and functionally active Schistosoma mansoni cathepsin B1 (SmCB1), or a cathepsin L from a related parasite Fasciola hepatica (FhCL1), elicits highly significant protection (up to $73 \%$ ) against an experimental challenge worm infection in murine models of schistosomiasis. The immune modulating properties of this subcutaneous injection can boost protection levels (up to 83\%) when combined with other S. mansoni vaccine candidates, glyceraldehyde 3-phosphate dehydrogenase (SG3PDH) and peroxiredoxin (PRX-MAP). Here, we discuss these data in the context of the parasite's biology and development, and provide putative mechanism by which the native-like cysteine peptidase induce protective immune responses.

Keywords: schistosome, cysteine peptidase, cathepsin B, Th2 immune response, papain
Schistosomiasis is caused by several helminth (worm) species of the genus Schistosoma, including Schistosoma mansoni, S. haematobium, and S. japonicum and is endemic in 74 tropical and sub-tropical countries, most prevalently in Africa, the Middle East, South America, and South-East Asia. Diagnostic tools for the detection of infection lack sensitivity and/or specificity leading the World Health Organization (WHO) to no longer provide estimates on populations infected or at risk but rather replacing this by describing the situation as "population requiring preventive chemotherapy." A recent WHO report (World Health Organization [WHO], 2012) revealed that the total number of people needing preventive chemotherapy globally for 2010 was over 237 million, of these $>108$ million were school-age children, and of which only $13 \%$ received treatment. Praziquantel is the only readily available effective drug for the treatment of the three main parasites causing human schistosomiasis, and has the advantages of low cost and self-limiting side effects. However, complete cure is seldom achieved such that for moderate (100-400 eggs per gram feces, epg) and heavy ( $>400$ epg) infections the cure rate may not exceed 60\%. Consequently, a substantial proportion of treated individuals can remain infected (probably unaware) and, therefore, are at risk of the serious sequelae of chronic schistosomiasis (Barsoum et al., 2013).
We cannot remain dependent on a single drug for the treatment and control of schistosomiasis as it is likely that in some time praziquantel-resistant parasites will emerge and, therefore, a vaccine for schistosomiasis is urgently needed. Schistosomes live intravascularly throughout their time spent in the mammalian host; the mature adult worms can reside in the mesenteric or pelvic veins for decades and are highly refractory to bloodborne immune defense elements. However, there is evidence that human populations can develop immune-mediated resistance to re-infection and, at least in animals, there is evidence that vaccine-related protective immune responses can be induced against the parasites. Schistosome larvae (cercariae) attenuated with gamma, X-ray, or ultraviolet radiation are capable of infecting their host through the skin but they do not survive to migrate beyond the lung stage; most are eliminated over a protracted time period of up to three weeks or more after infection (Harrop and Wilson, 1993). Laboratory animals immunized with radiation attenuated (RA) cercariae of S. mansoni and S. haematobium are protected against challenge infection with normal cercariae, with reductions in worm burdens compared to non-vaccinated animals varying from 30-90\% depending on the host, schistosome species and strain, numbers of immunizations, and time of challenge following immunization (Dean, 1983). Its proven efficacy in primates (Eberl et al., 2001; Kariuki et al., 2004) has reinforced 
the validity of the RA model, and it is still the gold standard against which the protective efficacies of recombinant antigens are compared.

Although there is no doubt that the immune response elicited by RA-vaccination mediates parasite attrition, there is no firm consensus on the phenotype of the protective immune response. Initial studies determined that reduction in challenge worm burden in RA-immunized mice correlated with polarized Th1 immune responses, characterized by the production of interferongamma (IFN- $\gamma$ ) by leukocytes in the airways of the lung (Smythies et al., 1992; Wilson et al., 1996). However, significant protection in the RA model was also found in $I L-12 p 40^{-/-}$mice (Anderson et al., 1998, 1999), and nitric oxide was shown not to be a major agent causing parasite elimination (Coulson et al., 1998). Furthermore, other studies have shown that effective immunity in the RA vaccine model involved Th2 (Mountford et al., 2001) or mixed Th1/Th2 (Hoffmann et al., 1999; Hewitson et al., 2005) immune responses. In contrast, studies on humans that exhibit resistance to infection following chemotherapy consistently indicate that Th2 immune responses correlate with protection (Ganley-Leal et al., 2006; Walter et al., 2006; McManus and Loukas, 2008; Jiz et al., 2009; Black et al., 2010a,b; Figueiredo et al., 2012; Fitzsimmons et al., 2012; Pinot de Moira et al., 2013; Wilson et al., 2014). It is possible that interpretation of these data is confounded by the role of T-cells in schistosome development. While the complete absence of $\mathrm{CD} 4^{+}$T-cells significantly impairs parasite growth and reproduction (Harrison and Doenhoff, 1983; Davies et al., 2001), so too does the suppression of Th2 immune responses during the pre-patent liver phase of infection (Riner et al., 2013). Both the lung and liver represent the major sites of attrition in RAimmunized mice challenged with S. mansoni (Laxer and Tuazon, 1992), suggesting that an alteration to the fine balance of Th1 and Th2 immune responses that occur in these anatomical sites at different times during natural infection may be sufficient to achieve protection, rather than a polarized response one way or the other.

What is clear is that irradiated cercariae elicit the production of IgG antibodies specific to parasite proteins, which induce protection when passively transferred to mice (Abath and Werkhauser, 1996). This correlates with the age-dependent development of human immunological resistance to reinfection with S. mansoni, which is also associated with the presence of anti-tegument IgG antibodies (Karanja et al., 2002). In addition, it has been proposed that the resistance to re-infection following chemotherapy with praziquantel, is mediated by antibodies specific to schistosome antigens released upon worm death that are not normally encountered by the host immune response (Mutapi et al., 1998; Gomes et al., 2002). These observations prompted searches to identify those molecules recognized by antibodies taken from RA cercariae-vaccinated mice, or humans resistant to re-infection. The most prominent of these were the tegument-associated antigens; Sm23 a member of the tetraspanin family of surface molecules (Da'dara et al., 2002, 2003), the fatty acid binding protein Sm14 (Tendler and Simpson, 2008) and the apical lipid bilayer-associated glucose transporter SGTP4 (Skelly et al., 1998; Krautz-Peterson et al., 2010). Despite the induction of parasite-specific cytokines and antibodies (Da'dara et al., 2002, 2003; Mahana, 2006), immunization with these antigens failed to elicit protection above the arbitrarily chosen 40\% threshold (Bergquist and Colley, 1998). Moreover, recent studies of human immune responses to these candidates did not pin-point any with a particular potential as a vaccine (Ribeiro de Jesus et al., 2000; Al-Sherbiny et al., 2003).

It is likely that antigens on the surface membrane of schistosome parasites are inaccessible to antibody binding (Keating et al., 2006; Tallima and El Ridi, 2008) which ensures that the antigens are protected behind a tight, almost impermeable, sphingomyelin-based hydrogen-bond network (Tallima and El Ridi, 2008; Migliardo et al., 2014). On the other hand, molecules released by the parasite during migration and development (termed excretory-secretory products; ESP) readily interact with specific antibodies and other effectors of the host defense system. Because the lung was demonstrated as the major site of attrition of schistosomes after immunization with RA vaccines, the early developing schistosome larvae are considered vulnerable targets of innate and adaptive immunity (Dean, 1983; Coulson, 1997). We have suggested that effective immune responses directed against these ESP harm the juvenile parasites as they pass through the narrow and convoluted capillaries of the lung (El Ridi et al., 2010). Therefore, the ESP represents a potential pool of vaccine targets. A number of ESP of cercariae, in vitrocultured and ex vivo lung-stage schistosomula, and adult worms of S. mansoni (Harrop et al., 1999; Knudsen etal., 2005; Curwen et al., 2006; Hansell et al., 2008; El Ridi and Tallima, 2009), S. japonicum (Liu et al., 2009; Liao et al., 2011) and S. haematobium (Young et al., 2012) have been identified; molecules common to these preparations include actin, heat shock proteins, enolase, aldolase, glutathione $S$-transferase, triose phosphate isomerase, glyceraldehyde 3-phosphate dehydrogenase (SG3PDH), 2 cisperoxyredoxin (PRX), and serine and, predominantly, cysteine peptidases.

Schistosomes express several different classes of cysteine peptidases. S. mansoni cathepsin B1 (SmCB1), a member of the lysosomal cysteine peptidases of the papain superfamily, was found to be expressed at high levels in the caecum and protonephridia of cercariae. Expression increases in the parasite gut soon after skin penetration and schistosomular transformation corresponding to the initiation of blood feeding (Zerda et al., 1988). The peptidase was reported to be the major hemoglobin-digesting enzyme alongside another papain-like cysteine peptidase, cathepsin L1 (SmCL1), both of which are major proteins in worm soluble extracts and ESP (Day et al., 1995; Dalton et al., 1996; Caffrey et al., 1997; Brady et al., 1999a,b, 2000; Bogitsh et al., 2001). SmCL1 efficiently degrades human hemoglobin to absorbable dipeptides and amino acids and is localized to the gastrodermis and to the tegument of adult worms. It displays $44 \%$ identity at the amino acid level with a second cathepsin L (SmCL2) which, by contrast, is not detected in cercarial extracts or gut caecum, but is predominantly localized to the reproductive system of the female parasite and to the gynecophoric canal of the male, implying involvement with the worm reproductive physiology (Dalton et al., 1996; Brady etal., 1999a,b, 2000; Stack et al., 2011). More recently, a third cathepsin L member, SmCL3, was shown to be expressed in 
the worm gastrodermis and was found to hydrolyze hemoglobin and serum albumin (Dvorák et al., 2009). Furthermore, a second cathepsin B (SmCB2) was localized to the schistosome tegument and may be involved in its biogenesis (Wippersteg et al., 2005).

Cathepsin B and cathepsin L activities are readily detected by enzymatic assays in S. mansoni cercarial extracts and may facilitate skin penetration (Dalton et al., 1997; Kasný et al., 2007, 2009; Dvorák et al., 2008). 8-10 day old cultured larvae of S. mansoni also exhibited a dramatic increase in expression of these hemoglobindegrading peptidases (Zerda et al., 1988; Dalton et al., 1995; Dalton and Brindley, 1996) and RNAi-mediated knockdown experiments showed that at least for SmCB1 the parasites cannot feed in vitro on hemoglobin and do not survive in vivo without this enzyme (Correnti et al., 2005). More recently, using microarray analysis, Gobert et al. (2010) showed that genes encoding cathepsin B and cathepsin L were greatly up-regulated in S. mansoni larvae cultured for 5 days (65 and 37-fold, respectively). Both SmCBs and SmCLs are highly immunogenic in infected mice and antibodies are detected in sera of $S$. mansoni-infected patients (Dalton et al., 1995; Dalton and Brindley, 1996; Planchart et al., 2007; Sulbarán et al., 2010).

The cathepsin B and L cysteine peptidases require the parasite gut-associated asparaginyl endopeptidase (Sm32) for their activation from an inactive zymogen to fully active mature enzyme (Dalton et al., 1995, 1996; Brindley et al., 1997; Skelly and Shoemaker, 2001; Sajid et al., 2003; Delcroix et al., 2006; KrautzPeterson and Skelly, 2008; Stack et al., 2011). Sm32 is also a cysteine peptidase, but is not a member of the papain superfamily (Dalton et al., 1995; Dalton and Brindley, 1996). Given their central importance in the biology of the parasite S. mansoni cysteine peptidases have been of interest as both vaccine candidates for disease prophylaxis and potential chemotherapeutic targets (Dalton and Brindley, 1996; Wasilewski et al., 1996; Abdulla et al., 2007).

In addition to their role in the biology of the parasite, we have shown that helminth cysteine peptidases have an ability to modulate the host immune response (O'Neill et al., 2000; Donnelly et al., 2010). Cysteine peptidases from such diverse sources as papaya (papain; Sokol et al., 2008), house dust mite (Derp1; Roche etal., 1997; Kikuchi etal., 2006), Leishmania mexicana (Pollock etal., 2003), and many fungal allergens (Shen et al., 1998; Kheradmand etal., 2002) have all been shown to skew the immune response toward a Th2 phenotype characterized by the release of IL-33 and IL-4 and the production of antigenspecific IgG1. Despite differences in amino acid sequence and tertiary structure, their shared ability to induce Th2 immune responses is dependent on their common enzymatic activity. Such is the potency of their ability to modulate immune responses that at low doses these cysteine peptidases can act as adjuvants, inducing Th2 responses to bystander antigens in the absence of another adjuvant (Chapman et al., 2007; Cunningham et al., 2012). Indeed, we have shown that the subcutaneous administration of papain prior to a challenge infection with S. mansoni, switched the parasite-specific immune response toward a Th2 phenotype. Furthermore, this approach resulted in a significant level of protection (50\%) from infection (El Ridi and Tallima, 2013).
The mechanism by which cysteine peptidase enzymes drive the amplification of Th2 immune responses is a subject of vigorous research. It has been suggested that proteases may degrade intracellular epithelia cell proteins, or damage epithelial cells, for example in the lung, which respond by secreting stressrelated cytokines including IL-12, IL-33, and thymic stromal lymphopoietin (TSLP), that subsequently activate mast cells and basophils (Tang et al., 2010; Liang et al., 2012). Innate lymphocytes, termed lung natural helper (LNH) cells, which produce IL-33 and TSLP, have also been implicated in papain-induced airway inflammation (Halim etal., 2012). Recent studies have indicated that a primary function of TSLP, produced as a result of protease activity, is to prevent the induction of Th1-inducing molecules such as IL-12 and CD70 from innate immune cells (Massacand etal., 2009), which in turn facilitates the development of Th2 immune responses. Interestingly, we found that, like papain, a vaccine formulation containing a combination of TSLP or IL-33 and larval ESP molecules reproducibly elicited production of parasite-specific Th2 cytokines and antibodies in response to a challenge infection with $S$. mansoni. Similar to the result achieved using papain, this vaccine formulation also elicited a highly significant $(P<0.0001)$ reduction of 62 (TSLP) to $78 \%$ (IL-33) in worm burden and worm egg load in host liver and small intestine (El Ridi and Tallima, 2013).

Indirectly, papain has been shown to mediate the differentiation of macrophages toward a Th2-associated M2 phenotype and via direct interaction, papain-treated macrophages are more likely to differentiate into M2 cells after stimulation with bacterial lipopolysaccharide, a ligand that more commonly induces inflammatory, Th1-associated M1 macrophages (Nhu et al., 2010, 2012). Consistent with these observations, we have previously reported that macrophages isolated from mice given a single parenteral injection of either SmCB1 or a Fasciola hepatica cathepsin L1 (FhCL1) were inhibited in their ability to produce Th1-inducing cytokines (Donnelly et al., 2010). The parasite proteases inhibited the TLR-TRIF dependent mechanism of activation by Th1associated ligands and thus we proposed that these macrophages were more permissive to stimulation with Th2-promoting ligands. In the absence of TLR3-dependent signaling, production of Th2 cytokines is significantly increased during a murine infection of S. mansoni (Joshi et al., 2008).

Therefore, we suggested that, like papain, SmCB1 could act as an adjuvant to elicit an antigen-specific Th2 immune response to co-injected parasite molecules. However, we also proposed that as immunization with SmCB1 would result in the production of cathepsin B-specific antibodies, it would likely elicit a higher level of protection than papain. To that end, SmCB1 was administered subcutaneously to outbred mice, which were then challenged with an infection of S. mansoni cercariae. Consistent with the data seen for papain and in agreement with our hypothesis, highly significant $(P<0.0001)$ and reproducible reduction of $50-70 \%$ in challenge worm burden was achieved in five consecutive experiments. Protection was associated with predominance of Th2-related cytokines and antibodies (El Ridi et al., 2014). However, we found that the reduction in worm egg counts in host liver and small intestine was not as striking 
as for the worm burden, in accordance with earlier findings correlating Th2 dominant responses with increased schistosome egg production (Wynn, 1999; Xu etal., 2009). Nevertheless, we observed that when a mixture of SmCB1 and FhCL1 were combined with the secreted proteins SG3PDH or SG3PDH and PRX-MAP a highly significant $(P<0.0001)$ and reproducible reduction of $66 \%$ in challenge worm burden and in worm egg load in liver and small intestine as well was achieved (El Ridi et al., 2014). The data confirmed that schistosome cysteine peptidases have in-built immune modulating properties that are protective on their own and have the potential to enhance the protective responses to other molecules. In line with all the evidence from allergen cysteine peptidases, this activity was related to their enzymatic activity since SmCB1 inactivated with inhibitors (E-64) or a non-active recombinant form of FhCL1 displayed markedly reduced level of Th2 mediators, and was associated with a significant decrease in protective capacity (El Ridi et al., 2014).

These results clearly demonstrate the induction of significant protection levels when an active parasite cysteine peptidase enzyme is used in the vaccine formulation with no additional requirement for an adjuvant. However, similar to the results achieved with the RA vaccination model, we have found inconsistencies between the characteristics of the antigen-specific T-cell response in protected animals. Instead, the key to the peptidasemediated protective effect may be the stimulation of a particular type of antibody response. Mathematical models have concluded that in younger, untreated endemic human populations, parasite infections activate short-lived plasma cells that are essentially non-protective. In contrast, in older populations the cumulative deaths of infection worms, due to natural death or chemotherapy, releases an antigen load which stimulates a different immune response, characterized by the production of long-lived plasma cells which reduces worm load (Mitchell et al., 2012). While it is clear that cysteine proteases, irrespective of their source, clearly induce the production of Th2-type antibodies against themselves and bystander antigens, there has been no investigation into the nature of antibody-producing B-cell. Recently, it was reported that immunization of mice with low doses of a fish venom protease (Natterin), induced the differentiation of terminally differentiated, long-lived antibody-secreting cells and that this was dependent on the proteolytic activity of the natterin (Komegae et al., 2013). In addition, these authors demonstrated that the production of both IL- 5 and IL-17 in response to the venom protease directly influenced the maintenance of the antibody secreting cells in the spleen (Grund et al., 2012). Therefore, its seems that the enzymatic activity of cysteine proteases, besides inducing antigen-specific cytokines, is also essential to generate survival signals necessary for the longevity of antibody secreting plasma cells.

Such potent, adjuvant-like effects of the cysteine peptidases may offer an innovative and feasible approach to developing a human vaccine formulation for protection against schistosomiasis. As we have learned, the delivery of parasite-secreted active cysteine peptidases alone or combined with other schistosome vaccine candidates, elicited levels of protection comparable to the bench-mark treatment of RA-cercariae. To date, the failure of many anti-schistosome vaccines has been attributed to the use of inappropriate adjuvants and/or delivery systems. Our data indicates that inclusion of active cysteine peptidases with inbuilt immunopotentiating activity in a vaccine preparation could preclude the need for a chemical adjuvant.

\section{REFERENCES}

Abath, F. G., and Werkhauser, R. C. (1996). The tegument of Schistosoma mansoni: functional and immunological features. Parasite Immunol. 18, 15-20. doi: 10.1046/j.1365-3024.1996.d01-6.x

Abdulla, M. H., Lim, K. C., Sajid, M., McKerrow, J. H., and Caffrey, C. R. (2007). Schistosomiasis mansoni: novel chemotherapy using a cysteine protease inhibitor. PLoS Med. 4:e14. doi: 10.1371/journal.pmed.0040014

Al-Sherbiny, M., Osman, A., Barakat, R., El Morshedy, H., Bergquist, R., and Olds, R. (2003). In vitro cellular and humoral responses to Schistosoma mansoni vaccine candidate antigens. Acta Trop. 88, 117-130. doi: 10.1016/S0001-706X(03)00195-5

Anderson, S., Shires, V. L., Wilson, R. A., and Mountford, A. P. (1998). In the absence of IL-12, the induction of Th1-mediated protective immunity by the attenuated Schistosome vaccine is impaired, revealing an alternative pathway with Th2-type characteristics. Eur. J. Immunol. 28, 2827-2838. doi: 10.1002/(SICI)1521-4141(199809)28:09<2827::AID-IMMU2827>3.0.CO;2-K

Anderson, S., Shires, V. L., Wilson, R. A., and Mountford, A. P. (1999). Formation of multi-nucleated giant cells in the mouse lung is promoted in the absence of interleukin-12. Am. J. Respir. Cell Mol. Biol. 20, 371-378. doi: 10.1165/ajrcmb.20.3.3317

Barsoum, R. S., Esmat, G., and El-Baz, T. (2013). Human schistosomiasis: clinical perspective. J. Adv. Res. 4, 433-444. doi: 10.1016/j.jare.2013.01.005

Bergquist, N. R., and Colley, D. G. (1998). Schistosomiasis vaccines: research to development. Parasitol. Today 14, 99-104. doi: 10.1016/S0169-4758(97)01207-6

Black, C. L., Muok, E. M., Mwinzi, P. N., Carter, J. M., Karanja, D. M., Secor, W. E., et al. (2010a). Increases in levels of schistosome-specific immunoglobulin $\mathrm{E}$ and $\mathrm{CD} 23(+) \mathrm{B}$ cells in a cohort of Kenyan children undergoing repeated treatment and reinfection with Schistosoma mansoni. J. Infect. Dis. 202, 399-405. doi: $10.1086 / 653828$

Black, C. L., Mwinzi, P. N., Muok, E. M., Abudho, B., Fitzsimmons, C. M., Dunne, D. W., et al. (2010b). Influence of exposure history on the immunology and development of resistance to human Schistosomiasis mansoni. PLoS Negl. Trop. Dis. 4:e637. doi: 10.1371/journal.pntd.0000637

Bogitsh, B. J., Dalton, J. P., Brady, C. P., and Brindley, P. J. (2001). Gut-associated immunolocalization of the Schistosoma mansoni cysteine proteases, SmCL1 and SmCL2. J. Parasitol. 87, 237-241. doi: 10.1645/00223395(2001)087[0237:GAIOTS]2.0.CO;2

Brady, C. P., Brindley, P. J., Dowd, A. J., and Dalton, J. P. (2000). Schistosoma mansoni: differential expression of cathepsins L1 and L2 suggests discrete biological functions for each enzyme. Exp. Parasitol. 94, 75-83. doi: 10.1006/expr.1999.4478

Brady, C. P., Dowd, A. J., Brindley, P. J., Ryan, T., Day, S. R., and Dalton, J. P. (1999a). Recombinant expression and localization of Schistosoma mansoni cathepsin L1 support its role in the degradation of host hemoglobin. Infect. Immun. 67, 368-374.

Brady, C. P., Dowd, A. J., Tort, J., Roche, L., Condon, B., O’Neill, S. M., et al. (1999b). The cathepsin L-like proteinases of liver fluke and blood fluke parasites of the trematode genera Fasciola and Schistosoma. Biochem. Soc. Trans. 27, 740-745.

Brindley, P. J., Kalinna, B. H., Dalton, J. P., Day, S. R., Wong, J. Y., Smythe, M. L., et al. (1997). Proteolytic degradation of host hemoglobin by schistosomes. Mol. Biochem. Parasitol. 89, 1-9. doi: 10.1016/S0166-6851(97)00098-4

Caffrey, C. R., Rheinberg, C. E., Moné, H., Jourdane, J., Li, Y. L., and Ruppel, A. (1997). Schistosoma japonicum, S. mansoni, S. haematobium, S. intercalatum, and S. rodhaini: cysteine-class cathepsin activities in the vomitus of adult worms. Parasitol. Res. 83, 37-41. doi: 10.1007/s004360050204

Chapman, M. D., Wünschmann, S., and Pomés, A. (2007). Proteases as Th2 adjuvants. Curr. Allergy Asthma Rep. 7, 363-367. doi: 10.1007/s11882-007-0055-6 Correnti, J. M., Brindley, P. J., and Pearce, E. J. (2005). Long-term suppression of cathepsin B levels by RNA interference retards schistosome growth. Mol. Biochem. Parasitol. 143, 209-215. doi: 10.1016/j.molbiopara.2005.06.007

Coulson, P. S. (1997). The radiation-attenuated vaccine against schistosomes in animal models: paradigm for a human vaccine? Adv. Parasitol. 39, 271-336. doi: 10.1016/S0065-308X(08)60048-2 
Coulson, P. S., Smythies, L. E., Betts, C., Mabbott, N. A., Sternberg, J. M., Wei, X. G., et al. (1998). Nitric oxide produced in the lungs of mice immunized with the radiation-attenuated schistosome vaccine is not the major agent causing challenge parasite elimination. Immunology 93, 55-63. doi: 10.1046/j.1365-2567.1998.00405.x

Cunningham, P. T., Elliot, C. E., Lenzo, J. C., Jarnicki, A. G., Larcombe, A. N., Zosky, G. R., et al. (2012). Sensitizing and Th2 adjuvant activity of cysteine protease allergens. Int. Arch. Allergy Immunol. 158, 347-358. doi: 10.1159/000334280

Curwen, R. S., Ashton, P. D., Sundaralingam, S., and Wilson, R. A. (2006). Identification of novel proteases and immunomodulators in the secretions of schistosome cercariae that facilitate host entry. Mol. Cell. Proteomics 5, 835-844. doi: 10.1074/mcp.M500313-MCP200

Da'dara, A. A., Skelly, P. J., Fatakdawala, M., Visovatti, S., Eriksson, E., and Harn, D. A. (2002). Comparative efficacy of the Schistosoma mansoni nucleic acid vaccine Sm23, following microseeding or gene gun delivery. Parasite Immunol. 24, 179 187. doi: 10.1046/j.1365-3024.2002.00453.x

Da'dara, A. A., Skelly, P. J., Walker, C. M., and Harn, D. A. (2003). A DNAprime/protein-boost vaccination regimen enhances Th2 immune responses but not protection following Schistosoma mansoni infection. Parasite Immunol. 25 429-437. doi: 10.1111/j.1365-3024.2003.00651.x

Dalton, J. P., and Brindley, P. J. (1996). Schistosome asparaginyl endopeptidase SM32 in hemoglobin digestion. Parasitol. Today 12, 125-129. doi: 10.1016/01694758(96)80676-4

Dalton, J. P., Clough, K. A., Jones, M. K., and Brindley, P. J. (1996). Characterization of the cathepsin-like cysteine proteinases of Schistosoma mansoni. Infect. Immun. 64, 1328-1334.

Dalton, J. P., Clough, K. A., Jones, M. K., and Brindley, P. J. (1997). The cysteine proteinases of Schistosoma mansoni cercariae. Parasitology 114, 105-112. doi: 10.1017/S003118209600830X

Dalton, J. P., Smith, A. M., Clough, K. A., and Brindley, P. J. (1995). Digestion of haemoglobin by schistosomes: 35 years on. Parasitol. Today 11, 299-303. doi: 10.1016/0169-4758(95)80045-X

Davies, S. J., Grogan, J. L., Blank, R. B., Lim, K. C., Locksley, R. M., and McKerrow, J. H. (2001). Modulation of blood fluke development in the liver by hepatic CD4+ lymphocytes. Science 294, 1358-1361. doi: 10.1126/science. 1064462

Day, S. R., Dalton, J. P., Clough, K. A., Leonardo, L., Tiu, W. U., and Brindley, P. J. (1995). Characterization and cloning of the cathepsin L proteinases of Schistosoma japonicum. Biochem. Biophys. Res. Commun. 217, 1-9. doi: 10.1006/bbrc. 1995.2737

Dean, D. A. (1983). Schistosoma and related genera: acquired resistance in mice. Exp. Parasitol. 55, 1-104. doi: 10.1016/0014-4894(83)90002-4

Delcroix, M., Sajid, M., Caffrey, C. R., Lim, K. C., Dvorák, J., Hsieh, I., et al. (2006). A multienzyme network functions in intestinal protein digestion by a platyhelminth parasite. J. Biol. Chem. 281, 39316-39329. doi: 10.1074/jbc. M607128200

Donnelly, S., O’Neill, S. M., Stack, C. M., Robinson, M. W., Turnbull, L., Whitchurch C., et al. (2010). Helminth cysteine proteases inhibit TRIF-dependent activation of macrophages via degradation of TLR3. J. Biol. Chem. 285, 3383-3392. doi: 10.1074/jbc.M109.060368

Dvorák, J., Mashiyama, S. T., Braschi, S., Sajid, M., Knudsen, G. M., Hansell, E., et al (2008). Differential use of protease families for invasion by schistosome cercariae. Biochimie 90, 345-358. doi: 10.1016/j.biochi.2007.08.013

Dvorák, J., Mashiyama, S. T., Sajid, M., Braschi, S., Delcroix, M., Schneider, E. L., etal. (2009). SmCL3, a gastrodermal cysteine protease of the human blood fluke Schistosoma mansoni. PLoS Negl. Trop. Dis. 3:e449. doi 10.1371/journal.pntd.0000449

Eberl, M., Langermans, J. A., Frost, P. A., Vervenne, R. A., Van Dam, G. J., Deelder, A M., et al. (2001). Cellular and humoral immune responses and protection against schistosomes induced by a radiation-attenuated vaccine in chimpanzees. Infect. Immun. 69, 5352-5362. doi: 10.1128/IAI.69.9.5352-5362.2001

El Ridi, R., and Tallima, H. (2009). Schistosoma mansoni ex vivo lung-stage larvae excretory-secretory antigens as vaccine candidates against schistosomiasis Vaccine 27, 666-673. doi: 10.1016/j.vaccine.2008.11.039

El Ridi, R., and Tallima, H. (2013). Vaccine-induced protection against murine schistosomiasis mansoni with larval excretory-secretory antigens and papain or type-2 cytokines. J. Parasitol. 99, 194-202. doi: 10.1645/GE-3186.1

El Ridi, R., Tallima, H., Mahana, N., and Dalton, J. P. (2010). Innate immunogenicity and in vitro protective potential of Schistosoma mansoni lung schistosomula excretory-secretory candidate vaccine antigens. Microbes Infect. 12, 700-709. doi: 10.1016/j.micinf.2010.04.012

El Ridi, R., Tallima, H., Selim, S., Donnelly, S., Cotton, S., Gonzales Santana, B., et al. (2014). Cysteine peptidases as schistosomiasis vaccines with inbuilt adjuvanticity. PLoS ONE 9:e85401. doi: 10.1371/journal.pone.0085401

Figueiredo, J. P., Oliveira, R. R., Cardoso, L. S., Barnes, K. C., Grant, A. V., Carvalho, E. M., et al. (2012). Adult worm-specific IgE/IgG4 balance is associated with low infection levels of Schistosoma mansoni in an endemic area. Parasite Immunol. 34, 604-610. doi: 10.1111/pim.12001

Fitzsimmons, C. M., Jones, F. M., Pinot de Moira, A., Protasio, A. V., Khalife, J., Dickinson, H. A., et al. (2012). Progressive cross-reactivity in IgE responses: an explanation for the slow development of human immunity to schistosomiasis? Infect. Immun. 80, 4264-4270. doi: 10.1128/IAI.00641-12

Ganley-Leal, L. M., Mwinzi, P. N., Cetre-Sossah, C. B., Andove, J., Hightower, A W., Karanja, D. M., et al. (2006). Correlation between eosinophils and protection against reinfection with Schistosoma mansoni and the effect of human immunodeficiency virus type 1 coinfection in humans. Infect. Immun. 74, 2169-2176. doi: 10.1128/IAI.74.4.2169-2176.2006

Gobert, G. N., Tran, M. H., Moertel, L., Mulvenna, J., Jones, M. K., McManus, D. P., et al. (2010). Transcriptional changes in Schistosoma mansoni during early schistosomula development and in the presence of erythrocytes. PLoS Negl. Trop. Dis. 4:e600 doi: 10.1371/journal.pntd.0000600

Gomes, Y. M., Pereira, V. R., Nakazawa, M., Montarroyos, U., Souza, W. V., and Abath, F. G. (2002). Antibody isotype responses to egg antigens in human chronic Schistosomiasis mansoni before and after treatment. Mem. Inst. Oswaldo Cruz 97(Suppl. 1), 111-112. doi: 10.1590/S0074-02762002000900022

Grund, L. Z., Komegae, E. N., Lopes-Ferreira, M., and Lima, C. (2012). IL-5 and IL-17A are critical for the chronic IgE response and differentiation of longlived antibody-secreting cells in inflamed tissues. Cytokine 59, 335-351. doi: 10.1016/j.cyto.2012.04.045

Halim, T. Y., Krauss, R. H., Sun, A. C., and Takei, F. (2012). Lung natural helper cells are a critical source of Th2 cell-type cytokines in protease allergen-induced airway inflammation. Immunity 36, 451-463. doi: 10.1016/j.immuni.2011. 12.020

Hansell, E., Braschi, S., Medzihradszky, K. F., Sajid, M., Debnath, M., Ingram, J., et al. (2008). Proteomic analysis of skin invasion by blood fluke larvae. PLoS Negl. Trop. Dis. 2:e262. doi: 10.1371/journal.pntd.0000262

Harrison, R. J., and Doenhoff, M. J. (1983). Retarded development of Schistosoma mansoni in immunosuppressed mice. Parasitology 86, 429-438. doi: $10.1017 /$ S0031182000050629

Harrop, R., Coulson, P. S., and Wilson, R. A. (1999). Characterization, cloning and immunogenicity of antigens released by lung-stage larvae of Schistosoma mansoni. Parasitology 118, 583-594. doi: 10.1017/S003118209900431X

Harrop, R., and Wilson, R. A. (1993). Irradiation of Schistosoma mansoni cercariae impairs neuromuscular function in developing schistosomula. J. Parasitol. 79, 286-289. doi: 10.2307/3283522

Hewitson, J. P., Hamblin, P. A., and Mountford, A. P. (2005). Immunity induced by the radiation-attenuated schistosome vaccine. Parasite Immunol. 27, 271-280. doi: 10.1111/j.1365-3024.2005.00764.x

Hoffmann, K. F., James, S. L., Cheever, A. W., and Wynn, T. A. (1999). Studies with double cytokine-deficient mice reveal that highly polarized Th1- and Th2-type cytokine and antibody responses contribute equally to vaccine-induced immunity to Schistosoma mansoni. J. Immunol. 163, 927-938.

Jiz, M., Friedman, J. F., Leenstra, T., Jarilla, B., Pablo, A., Langdon, G., et al. (2009). Immunoglobulin $\mathrm{E}$ ( $\mathrm{IgE}$ ) responses to paramyosin predict resistance to reinfection with Schistosoma japonicum and are attenuated by IgG4. Infect. Immun. 77, 2051-2058. doi: 10.1128/IAI.00012-09

Joshi, A. D., Schaller, M. A., Lukacs, N. W., Kunkel, S. L., and Hogaboam, C. M. (2008). TLR3 modulates immunopathology during a Schistosoma mansoni egg-driven Th2 response in the lung. Eur. J. Immunol. 38, 3436-3449. doi: 10.1002/eji.200838629

Karanja, K. M., Hightower, A. W., Colley, D. G., Mwinzi, P. N., Galil, K., Andove, J., et al. (2002). Resistance to reinfection with Schistosoma mansoni in occupationally exposed adults and effect of HIV-1 co-infection on susceptibility to schistosomiasis: a longitudinal study. Lancet 360, 592-596. doi: 10.1016/S0140-6736(02)09781-7

Kariuki, T. M., Farah, I. O., Yole, D. S., Mwenda, J. M., Van Dam, G. J., Deelder, A. M., et al. (2004). Parameters of the attenuated schistosome vaccine evaluated 
in the olive baboon. Infect. Immun. 72, 5526-5529. doi: 10.1128/IAI.72.9.55265529.2004

Kasný, M., Mikes, L., Dalton, J. P., Mountford, A. P., and Horák, P. (2007). Comparison of cysteine peptidase activities in Trichobilharzia regenti and Schistosoma man soni cercariae. Parasitology 134, 1599-1609. doi: 10.1017/S0031182007002910

Kasný, M., Mikes, L., Hampl, V., Dvorák, J., Caffrey, C. R., Dalton, J. P., et al. (2009). Peptidases of trematodes. Adv. Parasitol. 69, 205-297. doi: 10.1016/S0065308X(09)69004-7

Keating, J. H., Wilson, R. A., and Skelly, P. J. (2006). No overt cellular inflammation around intravascular schistosomes in vivo. J. Parasitol. 92, 1365-1369. doi: 10.1645/GE-864R.1

Kheradmand, F., Kiss, A., Xu, J., Lee, S. H., Kolattukudy, P. E., and Corry, D. B. (2002). A protease activated pathway underlying Th cell type 2 activation and allergic lung disease. J. Immunol. 169, 5904-5911. doi: 10.4049/jimmunol.169.10.5904

Kikuchi, Y., Takai, T., Kuhara, T., Ota, M., Kato, T., Hatanaka, H., et al. (2006). Crucial commitment of proteolytic activity of a purified recombinant major house dust mite allergen Der p1 to sensitization toward IgE and IgG responses. J. Immunol. 177, 1609-1617. doi: 10.4049/jimmunol.177.3.1609

Knudsen, G. M., Medzihradszky, K. F., Lim, K. C., Hansell, E., and McKerrow, J. H. (2005). Proteomic analysis of Schistosoma mansoni cercarial secretions. Mol. Cell. Proteomics 4, 1862-1875. doi: 10.1074/mcp.M500097-MCP200

Komegae, E. N., Grund, L. Z., Lopes-Ferreira, M., and Lima, C. (2013). The longevity of Th2 humoral response induced by proteases Natterins requires the participation of long-lasting innate-like B cells and plasma cells in spleen. PLoS ONE 8:e67135. doi: 10.1371/journal.pone.0067135

Krautz-Peterson, G., Simoes, M., Faghiri, Z., Ndegwa, D., Oliveira, G., Shoemaker, C. B., et al. (2010). Suppressing glucose transporter gene expression in schistosomes impairs parasite feeding and decreases survival in the mammalian host. PLoS Pathog. 6:e1000932. doi: 10.1371/journal.ppat.1000932

Krautz-Peterson, G., and Skelly, P. J. (2008). Schistosome asparaginyl endopeptidase (legumain) is not essential for cathepsin B1 activation in vivo. Mol. Biochem. Parasitol. 159, 54-58. doi: 10.1016/j.molbiopara.2007.12.011

Laxer, M. J., and Tuazon, C. U. (1992). Migration of 75Se-methionine-labeled Schistosoma japonicum in normal and immunized mice. J. Infect. Dis. 166, 1133 1138. doi: 10.1093/infdis/166.5.1133

Liang, G., Barker, T., Xie, Z., Charles, N., Rivera, J., and Druey, K. M. (2012). Naive T cells sense the cysteine protease allergen papain through protease-activated receptor 2 and propel TH2 immunity. J. Allergy Clin. Immunol. 129, 1377-1386.e13. doi: 10.1016/j.jaci.2012.02.035

Liao, Q., Yuan, X., Xiao, H., Liu, C., Lv, Z., Zhao, Y., et al. (2011). Identifying Schistosoma japonicum excretory/secretory proteins and their interactions with host immune system. PLoS ONE 6:e23786. doi: 10.1371/journal.pone.0023786

Liu, F., Cui, S. J., Hu, W., Feng, Z., Wang, Z. Q., and Han, Z. G. (2009). Excretory/secretory proteome of the adult developmental stage of human blood fluke, Schistosoma japonicum. Mol. Cell. Proteomics 8, 1236-1251. doi: 10.1074/mcp.M800538-MCP200

Mahana, N. A. (2006). Human and Murine Immune Responses to the Schistosoma mansoni Glucose Transporter. Ph.D. thesis, Faculty of Science, Cairo University, Giza.

Massacand, J. C., Stettler, R. C., Meier, R., Humphreys, N. E., Grencis, R. K., Marsland, B. J., et al. (2009). Helminth products bypass the need for TSLP in Th2 immune responses by directly modulating dendritic cell function. Proc. Natl. Acad. U.S.A. 106, 13968-13973. doi: 10.1073/pnas.0906367106

McManus, D. P., and Loukas, A. (2008). Current status of vaccines for schistosomiasis. Clin. Microbiol. Rev. 21, 225-242. doi: 10.1128/CMR.00046-07

Migliardo, F., Tallima, H., and El Ridi, R. (2014). Is there a sphingomyelin-based hydrogen bond barrier at the mammalian host-schistosome parasite interface? Cell Biochem. Biophys. 68, 359-367. doi: 10.1007/s12013-013-9716-3

Mitchell, K. M., Mutapi, F., Savill, N. J., and Woolhouse, M. E. (2012). Protective immunity to Schistosoma haematobium infection is primarily an anti-fecundity response stimulated by the death of adult worms. Proc. Natl. Acad. Sci. U.S.A. 109, 13347-13352. doi: 10.1073/pnas.1121051109

Mountford, A. P., Hogg, K. G., Coulson, P. S., and Brombacher, F. (2001) Signaling via interleukin-4 receptor alpha chain is required for successful vaccination against schistosomiasis in BALB/c mice. Infect. Immun. 69, 228-236. doi: 10.1128/IAI.69.1.228-236.2001

Mutapi, F., Ndhlovu, P. D., Hagan, P., Spicer, J. T., Mduluza, T., Turner, C. M., et al. (1998). Chemotherapy accelerates the development of acquired immune responses to Schistosoma haematobium infection. J. Infect. Dis. 178, 289-293. doi: $10.1086 / 517456$

Nhu, Q. M., Shirey, K. A., Pennini, M. E., Stiltz, J., and Vogel, S. N. (2012). Proteinaseactivated receptor 2 activation promotes an anti-inflammatory and alternatively activated phenotype in LPS-stimulated murine macrophages. Innate Immun. 18, 193-203. doi: 10.1177/1753425910395044

Nhu, Q. M., Shirey, K., Teijaro, J. R., Farber, D. L., Netzel-Arnett, S., Antalis, T. M., et al. (2010). Novel signaling interactions between proteinase-activated receptor 2 and Toll-like receptors in vitro and in vivo. Mucosal Immunol. 3, 29-39. doi: 10.1038/mi.2009.120

O’Neill, S. M., Brady, M. T., Callanan, J. J., Mulcahy, G., Joyce, P., Mills, K. H., et al. (2000). Fasciola hepatica infection downregulates Thl responses in mice. Parasite Immunol. 22, 147-155. doi: 10.1046/j.1365-3024.2000.00290.x

Pinot de Moira, A., Jones, F. M., Wilson, S., Tukahebwa, E., Fitzsimmons, C. M., Mwatha, J. K., et al. (2013). Effects of treatment on IgE responses against parasite allergen-like proteins and immunity to reinfection in childhood schistosome and hookworm coinfections. Infect. Immun. 81, 23-32. doi: 10.1128/IAI.00748-12

Planchart, S., Incani, R. N., and Cesari, I. M. (2007). Preliminary characterization of an adult worm "vomit" preparation of Schistosoma mansoni and its potential use as antigen for diagnosis. Parasitol. Res. 101, 301-309. doi: 10.1007/s00436007-0482-2

Pollock, K. G., McNeil, K. S., Mottram, J. C., Lyons, R. E., Brewer, J. M., Scott, P., et al. (2003). The Leishmania mexicana cysteine protease, CPB2.8, induces potent Th2 responses. J. Immunol. 170, 1746-1753. doi: 10.4049/jimmunol.170.4.1746

Ribeiro de Jesus, A., Araújo, I., Bacellar, O., Magalhães, A., Pearce, E., Harn, D., et al. (2000). Human immune responses to Schistosoma mansoni vaccine candidate antigens. Infect. Immun. 68, 2797-2803. doi: 10.1128/IAI.68.5.2797-2803.2000

Riner, D. K., Ferragine, C. E., Maynard, S. K., and Davies S. J. (2013). Regulation of innate responses during pre-patent schistosome infection provides an immune environment permissive for parasite development. PLoS Pathog. 9:e1003708. doi: 10.1371/journal.ppat.1003708

Roche, N., Chinet, T. C., and Huchon, G. J. (1997). Allergic and non-allergic interactions between house dust mite allergens and airway mucosa. Eur. Respir. J. 10, 719-726.

Sajid, M., McKerrow, J. H., Hansell, E., Mathieu, M. A., Lucas, K. D., Hsieh, I., et al. (2003). Functional expression and characterization of Schistosoma mansoni cathepsin B and its trans-activation by an endogenous asparaginyl endopeptidase. Mol. Biochem. Parasitol. 131, 65-75. doi: 10.1016/S0166-6851(03) 00194-4

Shen, H. D., Lin, W. L., Tam, M. F., Wang, S. R., Tsai, J. J., Chou, H., et al. (1998). Alkaline serine proteinase: a major allergen of Aspergillus oryzae and its crossreactivity with Penicillium citrinum. Int. Arch. Allergy Immunol. 116, 29-35. doi: $10.1159 / 000023921$

Skelly, P. J., and Shoemaker, C. B. (2001). Schistosoma mansoni proteases Sm31 (cathepsin B) and Sm32 (legumain) are expressed in the cecum and protonephridia of cercariae. J. Parasitol. 87, 1218-1221. doi: 10.1645/00223395(2001)087[1218:SMPSCB]2.0.CO;2

Skelly, P. J., Tielens, A. G., and Shoemaker, C. B. (1998). Glucose transport and metabolism in mammalian-stage schistosomes. Parasitol. Today 14, 402-406. doi: 10.1016/S0169-4758(98)01319-2

Smythies, L. E., Pemberton, R. M., Coulson, P. S., Mountford, A. P., and Wilson, R. A. (1992). T cell-derived cytokines associated with pulmonary immune mechanisms in mice vaccinated with irradiated cercariae of Schistosoma mansoni. J. Immunol. $148,1512-1518$.

Sokol, C. L., Barton, G. M., Farr, A. G., and Medzhitov, R. (2008). A mechanism for the initiation of allergen-induced T helper type 2 responses. Nat. Immunol. 9, 310-318. doi: 10.1038/ni1558

Stack, C., Dalton, J. P., and Robinson, M. W. (2011). The phylogeny, structure and function of trematode cysteine proteases, with particular emphasis on the Fasciola hepatica cathepsin L family. Adv. Exp. Med. Biol. 712, 116-135. doi: 10.1007/978-1-4419-8414-2_8

Sulbarán, G. S., Ballen, D. E., Bermúdez, H., Lorenzo, M., Noya, O., and Cesari, I. M. (2010). Detection of the Sm31 antigen in sera of Schistosoma mansoniinfected patients from a low endemic area. Parasite Immunol. 32, 20-28. doi: 10.1111/j.1365-3024.2009.01152.x

Tallima, H., and El Ridi, R. (2008). Schistosoma mansoni glyceraldehyde 3-phosphate dehydrogenase is a lung-stage schistosomula surface membrane antigen. Folia Parasitol. (Praha) 55, 180-186. doi: 10.14411/fp.2008.025 
Tang, H., Cao, W., Kasturi, S. P., Ravindran, R., Nakaya, H. I., Kundu, K., et al. (2010). The T helper type 2 response to cysteine proteases requires dendritic cellbasophil cooperation via ROS-mediated signaling. Nat. Immunol. 11, 608-617. doi: 10.1038/ni.1883

Tendler, M., and Simpson, A. J. (2008). The biotechnology-value chain: development of Sm14 as a schistosomiasis vaccine. Acta Trop. 108, 263-266. doi: 10.1016/j.actatropica.2008.09.002

Walter, K., Fulford, A. J., McBeath, R., Joseph, S., Jones, F. M., Kariuki, H. C., et al. (2006). Increased human IgE induced by killing Schistosoma mansoni in vivo is associated with pretreatment Th2 cytokine responsiveness to worm antigens. $J$. Immunol. 177, 5490-5498. doi: 10.4049/jimmunol.177.8.5490

Wasilewski, M. M., Lim, K. C., Phillips, J., and McKerrow, J. H. (1996). Cysteine protease inhibitors block schistosome hemoglobin degradation in vitro and decrease worm burden and egg production in vivo. Mol. Biochem. Parasitol. 81, 179-189. doi: 10.1016/0166-6851(96)02703-X

Wilson, R. A., Coulson, P. S., Betts, C., Dowling, M. A., and Smythies, L. E. (1996) Impaired immunity and altered pulmonary responses in mice with a disrupted interferon-gamma receptor gene exposed to the irradiated Schistosoma mansoni vaccine. Immunology 87, 275-282. doi: 10.1046/j.1365-2567.1996.465550.x

Wilson, S., Jones, F. M., Kenty, L. C., Mwatha, J. K., Kimani, G., Kariuki, H. C., et al. (2014). Post-treatment changes in cytokines induced by Schistosoma mansoni egg and worm antigens: dissociation of immunity and morbidity associated type-2 responses. J. Infect. Dis. doi: 10.1093/infdis/jit826 [Epub ahead of print].

Wippersteg, V., Sajid, M., Walshe, D., Khiem, D., Salter, J. P., McKerrow, J. H., et al. (2005). Biolistic transformation of Schistosoma mansoni with 5' flanking regions of two peptidase genes promotes tissue-specific expression. Int. J. Parasitol. 35, 583-589. doi: 10.1016/j.ijpara.2005.02.002

World Health Organization [WHO]. (2012). Weekly Epidemiological Record. 87(4):37-44. WHO Preventive Chemotherapy and Transmission Control Databank for Schistosomiasis. Available at: http://www.who.int/neglected_diseases/preventive_chemotherapy/sch/en/index.html
Wynn, T. A. (1999). Immune deviation as a strategy for schistosomiasis vaccines designed to prevent infection and egg-induced immunopathology. Microbes Infect. 1, 525-534. doi: 10.1016/S1286-4579(99)80092-6

Xu, X., Zhang, D., Sun, W., Zhang, Q., Zhang, J., Xue, X., et al. (2009). A Schistosoma japonicum chimeric protein with a novel adjuvant induced a polarized Th1 immune response and protection against liver egg burdens. BMC Infect. Dis. 9:54. doi: 10.1186/1471-2334-9-54

Young, N. D., Jex, A. R., Li, B., Liu, S., Yang, L., Xiong, Z., et al. (2012). Wholegenome sequence of Schistosoma haematobium. Nat. Genet. 44, 221-225. doi: 10.1038/ng.1065

Zerda, K. S., Dresden, M. H., and Chappell, C. L. (1988). Schistosoma mansoni: expression and role of cysteine proteinases in developing schistosomula. Exp. Parasitol. 67, 238-246. doi: 10.1016/0014-4894(88)90071-9

Conflict of Interest Statement: The authors declare that the research was conducted in the absence of any commercial or financial relationships that could be construed as a potential conflict of interest.

Received: 17 February 2014; paper pending published: 25 March 2014; accepted: 17 April 2014; published online: 08 May 2014.

Citation: El Ridi R, Tallima H, Dalton JP and Donnelly S (2014) Induction of protective immune responses against schistosomiasis using functionally active cysteine peptidases. Front. Genet. 5:119. doi: 10.3389/fgene.2014.00119

This article was submitted to Evolutionary and Genomic Microbiology, a section of the journal Frontiers in Genetics.

Copyright $\odot 2014$ El Ridi, Tallima, Dalton and Donnelly. This is an open-access article distributed under the terms of the Creative Commons Attribution License (CC BY). The use, distribution or reproduction in other forums is permitted, provided the original author(s) or licensor are credited and that the original publication in this journal is cited, in accordance with accepted academic practice. No use, distribution or reproduction is permitted which does not comply with these terms. 